\title{
Nonlinear Dynamics of a Bose-Einstein Condensate in a Magnetic Waveguide
}

\author{
H. Ott* J. Fortágh, S. Kraft, A. Günther, D. Komma, and C. Zimmermann \\ Physikalisches Institut der Universität Tübingen \\ Auf der Morgenstelle 14, 72076 Tübingen, Germany
}

(Dated: November 10, 2018)

\begin{abstract}
We have studied the internal and external dynamics of a Bose-Einstein condensate in an anharmonic magnetic waveguide. An oscillating condensate experiences a strong coupling between the center of mass motion and the internal collective modes. Due to the anharmonicity of the magnetic potential, not only the center of mass motion shows harmonic frequency generation, but also the internal dynamics exhibit nonlinear frequency mixing. Thereby, the condensate shows shape oscillations with an extremely large change in the aspect ratio of up to a factor of 10 . We describe the data with a theoretical model to high accuracy. For strong excitations we test the experimental data for indications of a chaotic behavior.

PACS numbers: 03.75.Kk, 03.75.Be, 05.45.-a
\end{abstract}

Since the first realization of Bose-Einstein condensates in 1995 ideas have been discussed how to exploit this new form of coherent matter for atom optics. Atom interferometry with single atoms has been extensively investigated in the past decade [1] and finds important applications for high precision measurements of gravitational or rotational forces 2, 3]. It was also possible to realize and demonstrate a number of atom optical elements such as mirrors [4], gratings [5], beamsplitters [6] and waveguides 7, 8]. It is a fascinating vision to integrate these elements on a microfabricated atom chip. Extremely sensitive chip based sensors for forces and accelerations as well as for applications in quantum computing are conceivable. Since Bose-Einstein condensates have been loaded into microfabricated traps [9, 10] this vision has changed into a very real research topic. Integrated atom optics with condensates combine the nonlinear interaction of a BoseEinstein condensates with trapping potentials, whose geometry is in general anharmonic. This is in contrast to both single atom experiments, where the interaction plays no important role, and conventional experimental arrangements for condensates, where the trapping potential is typically harmonic and consequently the center of mass (CM) motion is decoupled from the internal dynamics [1]. In microtraps one therefore expects not only a coupling between the external and internal dynamics but also pronounced nonlinear effects including even chaotic dynamics for large excitation amplitudes. The study of the dynamics of a condensate in micropotentials is an extension of previous work on collective excitations and provides essential information for future applications for integrated atom optics.

We present a detailed experimental study of the condensate dynamics in the most fundamental atom optical element - a magnetic waveguide. We regard waveguides which are closed at both ends such that the generic motion of the condensate is a periodic oscillation. The linear propagation of Bose-Einstein condensates along a non terminated guide was recently investigated in Refs. [13] and 14]. We have measured the time evolution of the CM motion and of the aspect ratio i. e. the ratio between the radial and the axial condensate radii. In contrast to previous experimental work on collective modes of a condensate, we realize a closed system with conserved energy and a self-sustained excitation scheme. This system allows us to realize high amplitude oscillations of the aspect ratio which exceed previously reported values by far. To describe the experimental data we have developed a theoretical model which is described in Ref. 15]. The model is based on the separation of the CM motion and reduces the internal dynamics to that of a condensate in a fictitious time-dependent harmonic potential. Such a scenario is known to be exactly solvable in ThomasFermi approximation [16, 17]. The model also allows us to identify an experimentally accessible regime where the dynamics become chaotic.

The waveguide potential is generated with microfabricated copper conductors at the surface of a chip. The chip is mounted upside down in a vacuum chamber at a background pressure of $10^{-11}$ mbar. The ${ }^{87} \mathrm{Rb}$ atoms are collected in a six-beam magneto-optical trap, pumped into the $\left(F=2, m_{\mathrm{F}}=2\right)$ ground state and trapped in a spherical magnetic quadrupole trap. The atoms are then adiabatically transferred into the waveguide potential at the chip surface by gradually changing the magnetic field geometry [9, 18]. By forced evaporation, Bose-Einstein condensates with 100.000 atoms are produced. For the presented experiments, the trapping frequencies are adjusted to $110 \mathrm{~Hz}$ in the radial and $8 \mathrm{~Hz}$ in the axial direction. The shape of the axial potential is sketched in Fig. 11. The waveguide potential is characterized by an almost constant radial oscillation frequency (the maximal deviations are smaller than 10 percent) and a strongly varying axial curvature. With a numerical calculation of the magnetic field, we determine the axial potential $U(x)$ to

$$
\frac{U(x)}{\mu}[G]=0.219 x^{2}-0.013 x^{3}-0.033 x^{4}-0.007 x^{5},
$$



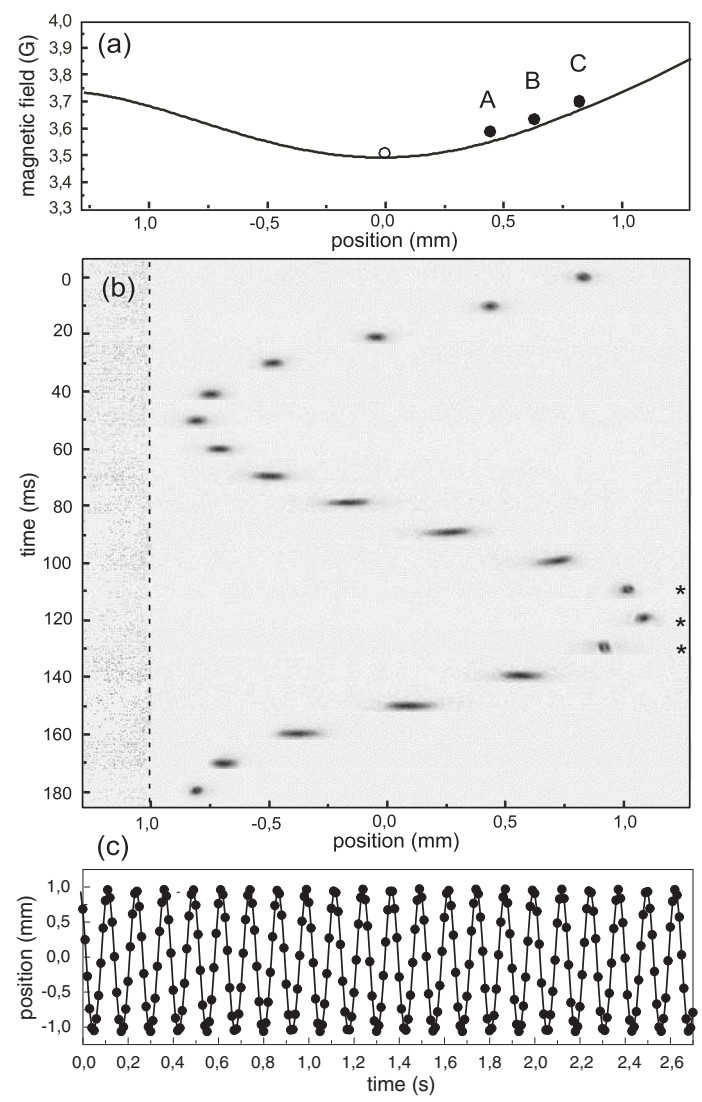

FIG. 1: Oscillation of the condensate in an anharmonic waveguide. The condensate is trapped below the microstructure which generates the magnetic field for the trapping potential. (a) Magnetic field in the trap minimum along the axial direction. The black dots indicate the starting positions of the oscillation for each experimental series. The circle marks the initial position without displacement. (b) Absorption images of the condensate after $20 \mathrm{~ms}$ time of flight (series $\mathrm{C}$, $10 \mathrm{~ms}$ intervals). Due to the time of flight, the amplitude of the oscillation is enhanced with respect to oscillation in the trap (also a phase shift occurs). For condensates marked with an asterisk the absorption images show diffraction and the aspect ratio can not be determined properly. (c) CM motion of the condensate (series B): experimental data (dots) and fit with three sinusoidal functions (line).

where $x$ has to be quoted in millimeter and $\mu$ is the magnetic moment of the atom. To induce the oscillation we displace the condensate within $400 \mathrm{~ms}$ with a magnetic field gradient and switch off the field gradient nonadiabatically $(<60 \mu \mathrm{s})$. The experiment was performed for three different initial displacements $d: 0.45 \mathrm{~mm}$ (series $\mathrm{A}$, duration $\left.t_{\mathrm{A}}=1.015 \mathrm{~s}\right), 0.63 \mathrm{~mm}\left(\mathrm{~B}, t_{\mathrm{B}}=2.7 \mathrm{~s}\right)$ and $0.79 \mathrm{~mm}\left(\mathrm{C}, t_{\mathrm{C}}=0.765 \mathrm{~s}\right)$. In Fig.10 the first 1.5 oscillation periods of series $\mathrm{C}$ are shown. To determine the $\mathrm{CM}$ position and the aspect ratio, the absorption images are fitted to a Thomas-Fermi profile. The aspect ratio bears the advantage of being independent from the
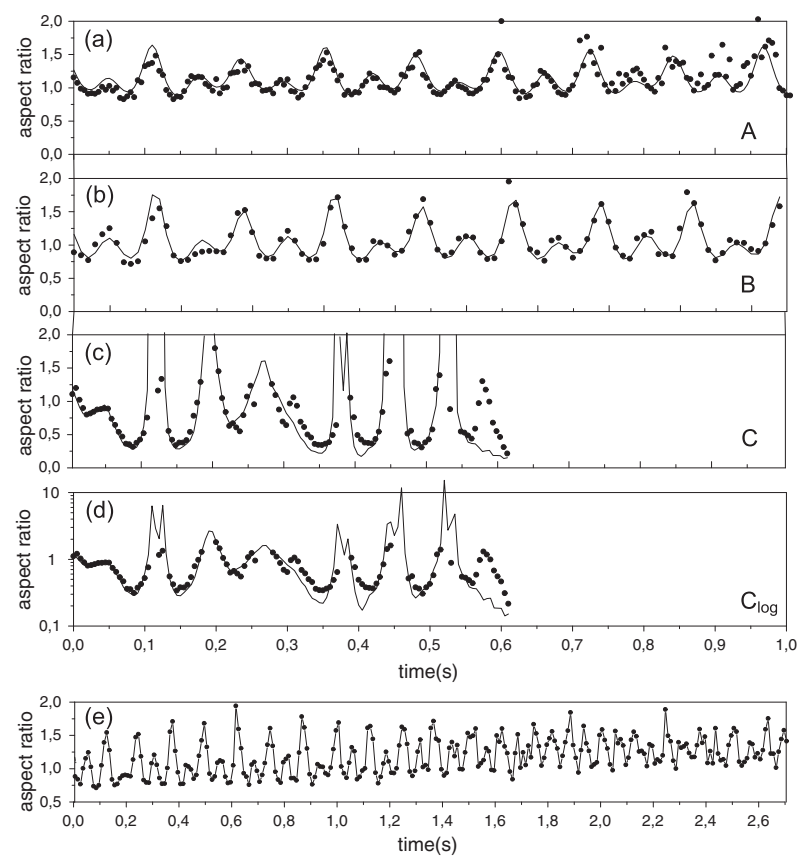

FIG. 2: Evolution of the aspect ratio of the condensate for the data series A (a), B (b), and C (c) (dots) and theoretical model (solid line). (d) Plot (c) with a logarithmic scale. (e) All data points of series B.

atom number. To exclude influences of the nearby copper conductors [19, 20], the experiment was carried out in a distance to the surface of $220 \mu \mathrm{m}$.

Our theoretical model describes the condensate with a set of two coupled differential equation systems. The first system are the classical equations of motion for the CM position of the condensate

$$
m \ddot{\mathbf{R}}=-\nabla U(\mathbf{R}),
$$

where $m$ is the mass of the atom, $\mathbf{R}$ is the CM position and $U$ is the confining potential. In Thomas-Fermi approximation, the density distribution of a condensate in a time-dependent harmonic potential can be written as follows [17]:

$$
n(\mathbf{x}, \mathbf{t})=\frac{\mu}{g} \frac{1}{\lambda_{1}(t) \lambda_{2}(t) \lambda_{3}(t)}\left(1-\sum_{i=1}^{3}\left(\frac{x_{i}}{r_{i 0} \lambda_{i}(t)}\right)^{2}\right),
$$

where $\mu$ is the chemical potential and $g$ is the coupling constant. The $\lambda_{i}(t)$ contain the complete time evolution of the Thomas-Fermi radii $r_{i}(t)=r_{i 0} \lambda_{i}(t)$. The $\lambda_{i}$ are governed by three ordinary differential equations

$$
\ddot{\lambda}_{i}=\frac{\omega_{i 0}^{2}}{\lambda_{i} \lambda_{1} \lambda_{2} \lambda_{3}}-\omega_{i}^{2}(t) \lambda_{i}, \quad i=1,2,3 .
$$

Here, $\omega_{i}(t)$ are the time-dependent oscillation frequencies of the harmonic potential. If the modulation of the 
potential starts at $t=0$, the initial conditions for the parameters are $\omega_{i}(0)=\omega_{i 0}$ and $r_{i}(0)=r_{i 0}$ with $\lambda_{i}(0)=1$. The anharmonicity of the potential now provides a coupling of the CM motion and the time evolution of the Thomas-Fermi radii, which is given by

$$
\omega_{i}^{2}(t)=\frac{1}{m} \frac{\partial^{2}}{\partial x_{i}^{2}} U(\mathbf{R}), \quad i=1,2,3 .
$$

To compare the experimental data with the model, (2), (4I) and (5) are numerically solved with the initial conditions $\lambda_{i}(0)=1$ and $\mathbf{R}(0)=(d, 0,0)$.

The experimental data for the CM motion of series B are presented in Fig. 15. We cannot extract any damping and the data are consistent with a $(1 / e)$ - lifetime of at least 20 minutes. Due to the anharmonicity of the potential (11), the frequency spectrum of the CM motion shows second and third harmonic generation. For series $\mathrm{C}$ with the largest initial displacement, the nonlinear effects are most pronounced and lead to a relative amplitude of 5 percent for the second harmonic and 1 percent for the third harmonic frequency with respect to the amplitude for the fundamental frequency $\nu_{0}$. For small amplitudes $\nu_{0}$ amounts to $8.5 \mathrm{~Hz}$ but is reduced with increasing displacement (A, B, and $\mathrm{C})$ by 4,6 , and 11 percent respectively. Because of the extremely low damping, the CM motion is suited to detect small forces, which may shift the trapping potential. An estimate for our experimental parameters indicates a resulting sensitivity of $F / m=10^{-4} \mathrm{~g}$, with the gravitational acceleration $g$.

In Fig. 2a -2 the measured aspect ratio is compared with the theoretical model. While the theory contains no free parameter, a constant multiplication factor of 1.2 is needed to describe the data accurately. This factor arises from the fact, that the condensate is radially compressed during the switch-off of the magnetic trap which - due to the mean field repulsion - leads to a larger radial size in the time-of-flight image 21]. The agreement between experiment and theory is remarkable and proves true the picture that the external motion in an anharmonic potential leads to a time-dependent harmonic potential in the rest frame. In Fig. 10, the condensates marked with an asterisk cannot be spatially resolved by our absorption imaging system, indicating an axial extension of the condensate of less than $15 \mu \mathrm{m}$. Therefore, the condensate may in fact follow the strong dynamics, which is predicted by the theory (Fig $2 \mathrm{~d})$. For the present data, we measure a change in the aspect ratio by more than a factor of 10 , the largest value reported so far. The data of series $\mathrm{B}$ which cover $2.7 \mathrm{~s}$ are promising to find effects arising from loss of atoms (Fig[2 $)$. At the end of the series, the atom number has dropped by more than a factor of 15 with only 3000 atoms left in the condensate. The systematic increase of the aspect ratio is due to the crossover to the $1 \mathrm{D}$ regime as defined in Ref. [22]. The Thomas-Fermi approximation breaks down because

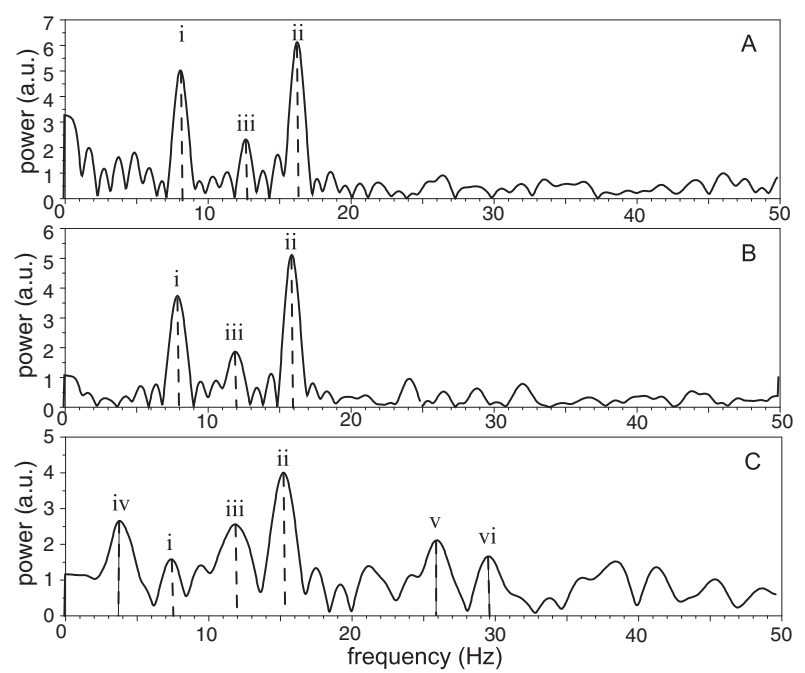

FIG. 3: Frequency spectra of the aspect ratio. The frequencies of the labeled peaks are: (i) fundamental frequency $\nu_{0}$, (ii) second harmonic frequency $2 \nu_{0}$, (iii) quadrupole resonance frequency $\nu_{q} \simeq \sqrt{5 / 2} \nu_{0}$, (iv) difference frequency $2 \nu_{0}-\nu_{q},(\mathrm{v})$ sum frequency $2 \nu_{0}+\nu_{q}$ and (vi) fourth harmonic frequency $4 \nu_{0}$.

the chemical potential of the condensate becomes smaller than the radial kinetic energy, which then dominates the radial expansion of the condensate.

We have performed a frequency analysis of the aspect ratio with an algorithm suitable for unequally spaced or missing data 23]. The results are shown in Fig. 3 The spectra show three major frequencies: the fundamental frequency $\nu_{0}$ (peak (i) in Fig.33), the second harmonic (ii) and the resonance frequency $\nu_{q}$ of the lowest lying collective mode (iii). The latter does not deviate more than 4 percent from its small amplitude value for strongly anisotropic traps which is given by $\sqrt{5 / 2} \nu_{0}$. Furthermore, the spectrum for series $\mathrm{C}$ (Fig. [3) shows several mixed frequencies. Two of them, the sum and the difference between the second harmonic frequency and the lowest resonance frequency $2 \nu_{0}-\nu_{q}$ and $2 \nu_{0}+\nu_{q}$ have been indentified ((iv) and (v)). This gives evidence for the nonlinear coupling of the collective excitations. Other than the coupled modes observed by Hechenblaikner et al. 24], the mode coupling in our experiment is nonresonant, and the mixed frequencies vanish in the limit of small amplitudes. Because the condensate performs a free oscillation in an external potential the total energy of the system is conserved. Summing up only the contributions for the internal energy of the condensate, one finds that the sum is not constant. Adding the kinetic and potential energy of the CM motion, the energy is conserved, which can be verified by using a Hamiltonian formalism [15]. Therefore, not only the internal dynamics is affected by the external motion, but there is also an energy exchange between the internal dynamics and the CM motion. In our experiment this effect leads to a 

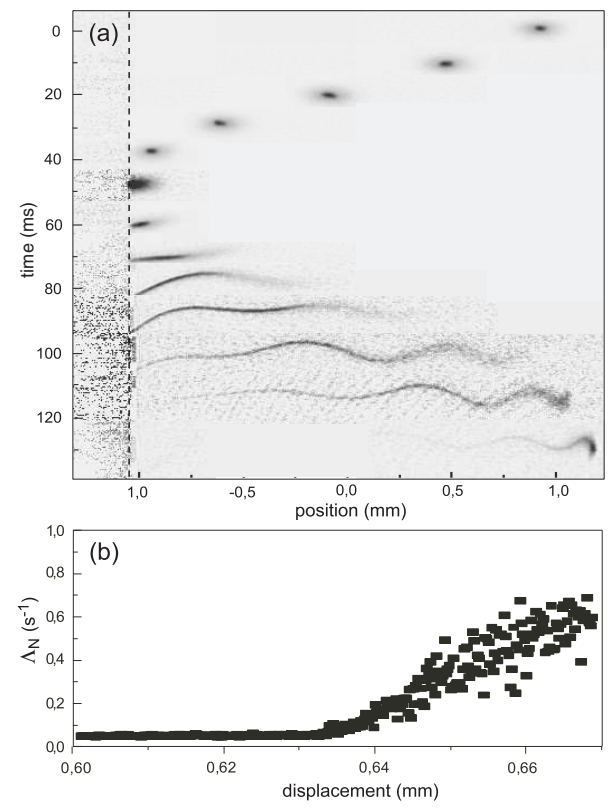

FIG. 4: (a) Oscillation for an initial displacement of $0.94 \mathrm{~mm}$. The absorption images are taken after $20 \mathrm{~ms}$ time of flight. The vertical dotted line indicates the surface of a wire which narrows the display window. (b) Lyapunov exponent: $\Lambda_{\mathrm{N}}=$ $1 /(N \tau) \sum_{i=1}^{N} \ln d_{i} / d_{0}$ as a function of the initial displacement $d$. $d_{0}$ is the initial Euclidian distance between two neighboring trajectories, $\tau$ is the duration of one iteration and $N$ is the number of iterations. The Lyapunov exponent is defined in the limit $\Lambda=\lim _{N \rightarrow \infty} \Lambda_{\mathrm{N}}$. Each pair of trajectories was numerically solved for $200 \mathrm{~s}\left(d_{0}=0.1 \mu \mathrm{m}, \tau=1 \mathrm{~ms}, N=\right.$ $2 \times 10^{5}$ ). Above a displacement of $0.67 \mathrm{~mm}$ the numerics are no longer stable for the total propagation time.

theoretically calculated amplitude variation for the CM motion of $1 / 4000$, which is too small to be detected.

Fig. 4a shows absorption images for an initial displacement of $0.94 \mathrm{~mm}$. The data show a strong axial excitation of the condensate, which raises the question of the onset of chaos. A signature of a chaotic behavior in experimental data is an enhanced noise in the frequency spectrum of the data. The spectrum of the aspect ratio for series $\mathrm{C}$ in Fig. 3 clearly shows an enhanced background, compared to the two other series. However, this should not be regarded as an unchallengeable experimental proof. Such a proof would require a longer data set and an enhanced resolution of the imaging system. Nevertheless, the theoretical model can be analyzed for the experimental parameters. One of the strongest evidences for a chaotic dynamic is a positive Lyapunov exponent, which indicates an exponential sensitivity to the initial conditions. For this purpose neighboring trajectories for slightly different initial displacements have been simulated over $200 \mathrm{~s}$. Fig. 4 shows the evolution of the Lyapunov exponent with increasing displacement. For a displacement of more than $0.63 \mathrm{~mm}$, the calculated Lya- punov exponent becomes positive and grows linearly. As the total energy of the system is conserved, one can easily test the numerical stability and exclude any numerical artefacts. The corresponding Poincaré maps and the frequency spectra of the trajectories indicate the same results. This gives strong evidence, that series $\mathrm{C}$ was performed in a regime where the longtime behavior of the trajectory is chaotic.

In conclusion, we have studied the dynamics of a BoseEinstein condensate oscillating in an anharmonic trapping potential. We have identified several nonlinear effects including second and third harmonic generation of the center of mass motion, a coupling of the internal and external dynamics and nonlinear mode mixing. The theoretical model describes these effects with high accuracy. The model predicts a chaotic dynamic above a critical oscillation amplitude and the experimental data give indication for a chaotic motion too. Considering applications of magnetic microtraps and integrated atom optics, we have shown that the motion of a condensate in a magnetic waveguide necessarily leads to excitations of the inner degrees of freedom. A thorough understanding of these effects plays a key role to exploit the nonlinearity to build high precision sensors. It also shows fundamental limits for the use of Bose-Einstein condensates for technical applications.

We gratefully acknowledge financial support from the Deutsche Forschungsgemeinschaft under Grant No. Zi/419-5.

* Electronic address: ott@pit.physik.uni-tuebingen.de

[1] Atom Interferometry, edited by Paul Berman (Academic Press, Chestnut Hill, MA, 1997).

[2] F. Riehle et al., Phys. Rev. Lett. 67, 177 (1991).

[3] M. Kasevich and S. Chu, Phys. Rev. Lett. 67, 181 (1991).

[4] K. S. Johnson et al., Phys. Rev. Lett. 81, 1137 (1998).

[5] E. M. Rasel et al., Phys. Rev. Lett. 75, 2633 (1995).

[6] D. Cassettari et al., Phys. Rev. Lett. 85, 5483 (2000).

[7] M. Key et al., Phys. Rev. Lett. 84, 1371 (2000).

[8] D. Müller et al., Phys. Rev. Lett. 83, 5194 (1999).

[9] H. Ott et al., Phys. Rev. Lett. 87, 230401 (2001).

[10] W. Hänsel et al., Nature 413, 498 (2001).

[11] J. F. Dobson, Phys. Rev. Lett. 73, 2244 (1994).

[12] L. Deng et al., Nature 398, 218 (1999).

[13] A. E. Leanhardt et al., Phys. Rev. Lett. 89, 040401 (2002)

[14] J. Fortágh et al., to be published in Appl. Phys. B

[15] H. Ott et al., cond-mat/0212193

[16] Y. Kagan, E. L. Surkov, and G. V. Shlyapnikov, Phys. Rev. A 55, R18 (1997).

[17] Y. Castin and R. Dum, Phys. Rev. Lett. 77, 5315 (1996).

[18] J. Fortágh et al., Appl. Phys. Lett. 81, 1146 (2002).

[19] J. Fortágh et al., Phys. Rev. A 66, 041604(R) (2002)

[20] S. Kraft et al., J. Phys. B: At. Mol. Opt. Phys. 35, L469 (2002).

[21] Assuming a stepwise change of the radial trapping fre- 
quency we can include the distortion in the theory to model the effect of the compression. We find e.g., that an increase of the radial trapping frequency to $500 \mathrm{~Hz}$ for a duration of $70 \mu \mathrm{s}$ is sufficient to cause the factor of 1.2 .
[22] A. Görlitz et al., Phys. Rev. Lett. 87, 130402 (2001).

[23] N. R. Lomb, Astrophys. Space Sci. 39, 447 (1976).

[24] G. Hechenblaikner et al., Phys. Rev. Lett. 85, 692 (2000). 